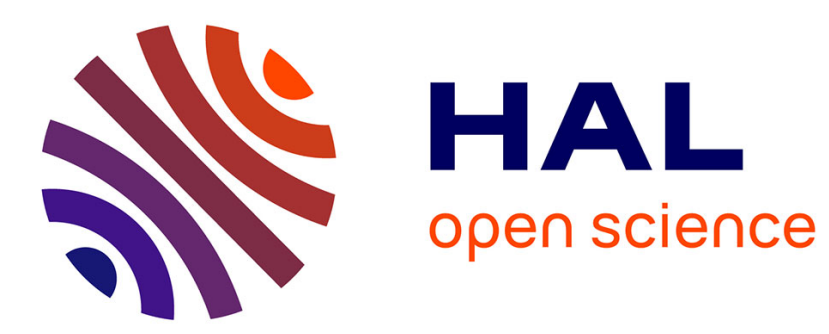

\title{
Cuckoo Scheduling Algorithm for Lifetime Optimization in Sensor Networks of IoT
}

\author{
Mazin Kadhum Hameed, Ali Kadhum Idrees
}

\section{To cite this version:}

Mazin Kadhum Hameed, Ali Kadhum Idrees. Cuckoo Scheduling Algorithm for Lifetime Optimization in Sensor Networks of IoT. Inventive Systems and Control, 204, Springer Singapore, pp.171-187, 2021, Lecture Notes in Networks and Systems, 10.1007/978-981-16-1395-1_14 . hal-03399746

\section{HAL Id: hal-03399746 \\ https://hal.science/hal-03399746}

Submitted on 24 Oct 2021

HAL is a multi-disciplinary open access archive for the deposit and dissemination of scientific research documents, whether they are published or not. The documents may come from teaching and research institutions in France or abroad, or from public or private research centers.
L'archive ouverte pluridisciplinaire HAL, est destinée au dépôt et à la diffusion de documents scientifiques de niveau recherche, publiés ou non, émanant des établissements d'enseignement et de recherche français ou étrangers, des laboratoires publics ou privés. 


\title{
Cuckoo Scheduling Algorithm for lifetime optimization in Sensor Networks of IoT
}

Mazin Kadhum Hameed ${ }^{1}$ and Ali Kadhum Idrees ${ }^{2 .}$

${ }^{1}$ Department of Software, University of Babylon, Babylon, Iraq.

1it.mazen.kadhum@uobabylon.edu.iq

2 Department of Computer Science, University of Babylon, Babylon, Iraq

2,* ali.idrees@uobabylon.edu.iq

* Corresponding Author

\begin{abstract}
The network topology control represents an essential factor during designing the Wireless Sensor Networks (WSNs) due to its primary role in the lifetime optimization of the WSNs. This paper proposes a Cuckoo Scheduling Algorithm (CSA) for lifetime optimization in Sensor Networks of Internet of Things (IoT). First, the sensor devices have clustered using the DBSCAN method, and then the CAS technique is applied at each cluster head. The CSA provides the best schedule of the sensor nodes inside each cluster to monitor the cluster region of with a minimum number of nodes while keeping a suitable level of coverage. The cluster head pooling and the CSA have executed periodically. The experimental results confirm that the CSA technique enhances the network lifespan and whilst preserving an acceptable ratio of coverage in WSNs.
\end{abstract}

Keywords: Sensor Networks, DBSCAN clustering, Cuckoo Algorithm, performance evaluation, Energy saving.

\section{Introduction}

Sensors could be defined as small, economizing, and low-power operating devices that can sense, process, and transmit any acquired data over wireless networks. The WSN are an essential factor in a wide variety of uses, including security, battlefield surveilling, air traffic controlling, bio-detection, environmental monitors, industrial automation, and smart grids [1, 2]. Monitor such uses demands the deployment of several sensors in the environment. The area that could be reached by the sensor during monitoring or sensing is known as its coverage, as a sensor is capable of monitoring multiple targets in its coverage [3].

The relatively smaller size of sensors, as well as their uses in hardly accessible regions make it rather impractical to replace or change their batteries [4]. Therefore, expanding the network lifespan is considered to be an important issue in Wireless Sensor Networks. Among the main aspects to be taken into consideration when 
increasing the lifespan are deploying sensors optimally [5-7], sleep scheduling (i.e.) changing the sensor mode from active to sleep [8-11], and maintaining the balance of load [12].

Hence, an efficient use of the energy could be realized by relying on the scheduling of sensor nodes for monitoring the target, as this would help in avoiding network congestions and packet retransmitting, thereby reducing any additional energy consumption for communication within networks [13]. As for the target coverage, the sensor scheduling remarkably extends the network lifespan. Multiple sensors could be clustered in sensor sets, which could either have a duplicate or not. Whenever such a set is in the active mode, the element sensor node may be active or inactive, meanwhile the remainder (the elements of other sets) are idle. Maintaining the balance of load represents another essential variable in achieving a maximal network lifespan. The extent to which the energy is available for every sensor differs remarkably according to its distance from the base station as well as its sense range and the quantity of the node's transmitting power consumption. According to the usable residual energy, a balance could be drawn among sensor loads $[14,15]$.

This paper introduces the following contributions

i) This paper suggests Cuckoo Scheduling Algorithm (CSA) for Enhancing Lifetime of Cluster-based WSNs. This technique achieves two efficient algorithms: clustering and then scheduling the sensor devices in WSN. First, the WSN is clustered into clusters using the distributed DBSCAN approach. The scheduling phase splits the lifespan into periods, and it achieves its goal by three steps. In the first step, the cluster head polling is implemented in a distributed way inside each cluster. After that, the cluster head will execute Cuckoo Algorithm in the second step to optimize the coverage and the lifespan of the network to produce the optimal schedule of sensor devices that are responsible for monitoring the cluster region in the next step. In the fourth step, each sensor device will receive a packet from the cluster head to inform it to stay active or sleep until the beginning of the next period.

ii) The Cuckoo Algorithm (CA) is employed to optimize the network lifespan while maintaining acceptable coverage for the monitoring area. CA is applied to solve the proposed model of optimization to reduce the centers' number of devices that are not covered and decrease the number of the active sensor device in each period. The scheduling algorithm-based CA is to provide the best schedule of sensor devices in each period instead of using optimization solvers. This can decrease the execution time and maximize network lifespan. 
iii) Extensive experiments are performed using the $\mathrm{C}++$ custom simulator. The conducted simulation results prove that the proposed CSA technique can improve the lifespan of the network and maintaining an acceptable level of coverage in the area of interest in comparison with some existing methods such as DESK [26], GAF [25], and PeCO [49].

The remainder of the work could be sketched as follows: Section 2 discusses the related works, Section 3 reviews the CS and DBSCAN algorithms, and Section 4 presents a description of the proposed algorithm. Sections 5 and 6 present and discuss the results of experiment and concluding remarks, respectively.

\section{Related Works}

Yaxiong and Jie (2010) introduced a generic duty-cycling scheduling technique that depends on the stochastic theory[16]; whereas Chih-fan and Mingyan (2004) proposed a precise scheduling algorithm as part of every approach, along with the analysis of the coverage and duty cycle characteristics[17]. Both Nath and Gibbons (2007) and Zhuxiu et al, created two common Sleep Scheduling algorithms, further identified as the Connected K-Neighbourhood (CKN) and the enhanced Energy Consumed uniformly - Connected K-Neighbourhood (EC-CKN) algorithms, respectively [18]. Any of these two scheduling algorithms could close the lower power nodes meanwhile each of the network's connectivity and its sufficient routing latency are maintained. As for the $\mathrm{CKN}$ algorithm, every node is $\mathrm{K}$ connected, implying that whenever a node has over $\mathrm{K}$ active neighbours (the k-connectivity is satisfied), it will make the decision of closing itself, otherwise (if the active adjacent neighbours are under $\mathrm{K}$ ), the node will remain active. Being a distributed SS algorithm, it could efficiently extend the lifespan of every single node and thereby eventually the whole network's lifespan. The rank value could be used in identifying the active nodes within CKN algorithms. The initial phase of performing CKN algorithms is provided for every period in a random manner, where the set of active nodes differs from one period to another. Yet, the main issue of this type of algorithms lies in the fact that their energy could not be guaranteed to have a uniform consumption [19]. Alternatively, the EC-CKN algorithm considers the remainder of the energy of for the nodes based on the CKN, thereby balancing the amount of energy consumed by the network as a whole and at the same time keeping it K-connected.

In [20] a scheduling technique is suggested according to generic duty-cycling by use of the stochastic theory, meanwhile [21] introduced the coverage and duty cycle feature analyses that are determined by scheduling algorithms. Based on 
where the neighbouring nodes are located, Sun et al. [22] employed redundancy algorithms in judging the sensor node status, as any redundant node will be turned into sleep mode.The Sleep-awake Energy Efficient Distributed (SEED) algorithm was introduced by Ahmed et al [23]. It divides any network field into three different regions; the high-energy region cluster heads take the responsibility of communicating with the base station and conserving the energy of low energy region cluster heads. The SEED outperformed various previously proposed energyefficient mechanisms in the field.

The work in [26] proposed a scheduling algorithm that can schedule the sensor devices in subdivided grids of the area of interest based on their geographical positions of sensor devices. A distributed scheduling algorithm named DESK is proposed by [25]. The authors executed the DESK algorithm at each sensor device and the decision is based on local information from the neighbouring devices using perimeter coverage model. The authors in [27, 28] proposed two coverage algorithms to extend the lifespan of the WSNs. The first algorithm is named DiLCO that use an optimization solver to a coverage optimization model based on primary points to produce the optimal schedule in ach round. The second algorithm proposed an optimization model for maintain the coverage and improve the lifespan of the WSNs based on the perimeter coverage model.

Several algorithms have proposed to solve the scheduling problem in WSNs. Some of them distributed scheduling approaches, which are fast because they have based on local information, but they cannot give an optimal schedule of sensor devices. The other types of scheduling algorithms are centralized, and they can provide an optimal solution but with a high execution time in the case of large WSN. Some proposed works proposed scheduling algorithms that are globally distributed but locally centralized and using optimization solvers. These methods can enhance network lifespan, but they consume a high execution time.

In this paper, a Cuckoo Scheduling Algorithm (CSA) for Improving the lifespan of Cluster-based WSNs. This technique partitions the WSN lifespan into periods. Every period performs a distributed network lifespan optimization based on distributed DBSCAN clustering, distributed cluster head polling, and sensor scheduling based on CA. This approach extends the network lifespan while maintaining a suitable level of coverage for the monitored area of interest. 


\section{Proposed CSA technique}

In this article, a Cuckoo Scheduling Algorithm for Enhancing Lifespan of Cluster based WSNs, called (CSA) technique is suggested. It is composed of two phases: Clustering and scheduling. Figure 1 illustrates the proposed CSA technique.

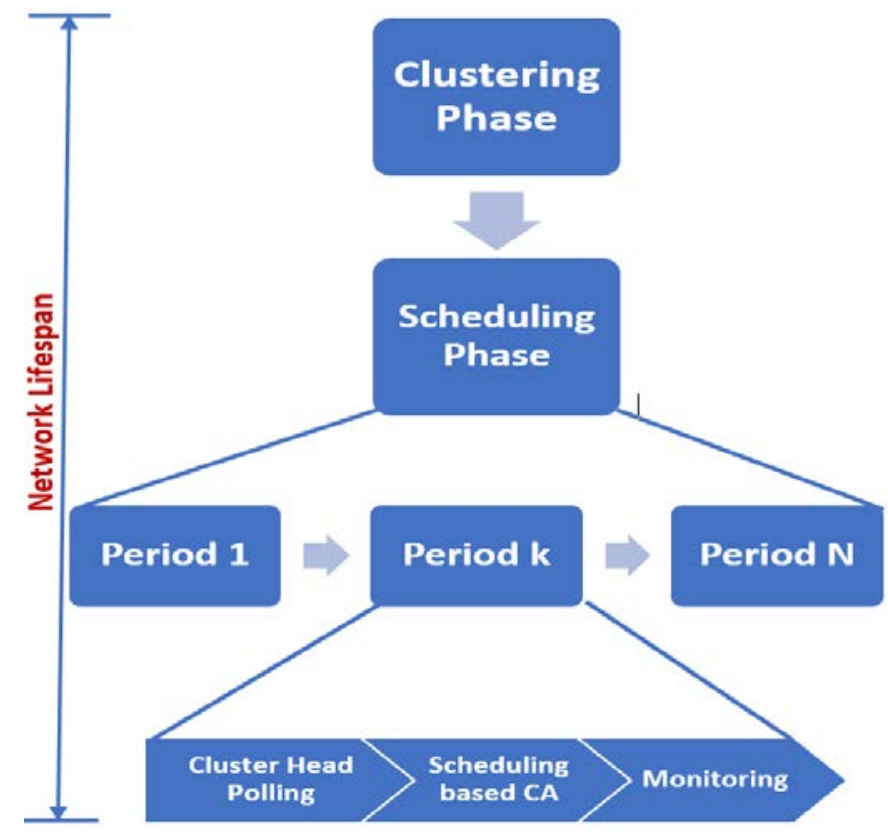

Figure 1 CSA technique.

\subsection{Clustering phase}

The sensor devices in the network are clustered based on our proposed distributed DBSCAN clustering algorithm introduced in [29]. This algorithm will group the nodes into clusters, after that the nodes in each cluster cooperate and share their information to each other for choosing a cluster head periodically.

This algorithm is selected for the follwing different reasons:

1. DBSCAN does not require one to specify the number of clusters in the data a priori, as opposed to k-means.

2. DBSCAN can find arbitrarily-shaped clusters. It can even find a cluster completely surrounded by (but not connected to) a different cluster.

3. DBSCAN has a notion of noise, and is robust to outliers. 
4. The parameters minPts and $\varepsilon$ can be set by a domain expert, if the data is well understood.

According to the Dbscan algorithm, the algorithm for each sensor to execute DbScan is as follows:

- According to the principle of the Dbscan algorithm, each sensor node will perform the same test, regardless of whether it is a core point, because it scans the surrounding area to find out the number of sensors that are within the sensing range and must be larger or equal to specific parameters.

- For sensor nodes within the sensing range of the core point, they will become its members.

- If this core point does not belong to any cluster, it will form a new cluster, otherwise it will remain in the same cluster.

- The core point sends a message to all its members to be included in the same cluster.

- Repeat steps 1-5 until all sensor nodes pass.

Algorithm 2. Distributed DBSCAN (sj)

Input: N: number of neighbor nodes, Sr: sensing range, minNodes: minimum number of nodes to create cluster.

Output: sj.rejon: the cluster number for node sj.

1: $\quad$ while $R E j \geq$ Ethr do

2: If sj Receive MemberPacket from si then

3: $\quad$ Mark sj as member to the Core si ;

4: $\quad$ Update REj;

5: $\quad$ end

6: $\quad$ sj.rejon $\leftarrow 0$;

7: $\quad$ for each node si in $\mathbf{N}$ do $/ / \mathbf{i} \in \mathbf{N}$ and $\mathbf{i} \neq \mathbf{j}$

8: $\quad$ nbrNodes $\leftarrow$ nbrNodes + CORE Objective Function (sj, si, Sr);

9: $\quad$ if CORE Objective Function return 1 then

10: $\quad$ Send MemberPacket to the sensor node $i$;

11: Update REj;

12: $\quad$ end

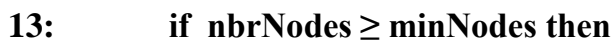

14: save the information

15: if $((($ sj.rejon $=0)$ Or $($ sj.rejon $\neq 0))$ and $(r==0))$ then

16: $\quad$ sj.rejon $\leftarrow$ sj.rejon +1 ;

17: Call Cluster(sj);

18: $\quad$ end

19: $\quad$ else if $(($ sj.rejon $=0)$ Or $(r \neq 0))$ then 


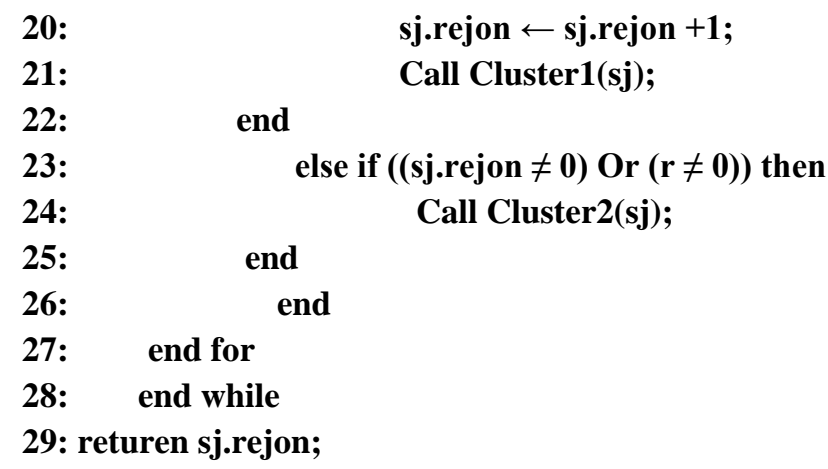

CORE Objective Function return 1 and $r=\mathbf{0}$ if the sensor node $i$ is within the sensing range $\mathrm{Sr}$ and it isn't always a member in other clusters. Otherwise, CORE Objective Function returns $\mathbf{0}$ and $\mathrm{r}=1$. The function Cluster placed any neighbor node inside the sensing variety of sj in the same cluster and sj send MemberPacket to the sensor node i to inform it that it turns into a member in the identical cluster of sj. The function Cluster1 placed any neighbor node in the sensing variety of sj and it has no longer assigned to any cluster in the identical cluster of sensor node $\mathrm{j}$. The function Cluster2 positioned any neighbor node within the sensing range of sj and it has no longer assigned to any cluster (or it is assigned to the cluster of sensor node $\mathrm{j}$ ) in the same cluster of sensor node $\mathrm{j}$. After reaching the capabilities Cluster, Cluster1, and Cluster2, the remaining electricity of the sensor node j might be updated because of sending a MemberPacket to the sensor node i to inform it that it becomes a member in the equal cluster of $\mathrm{sj}$.

\subsection{Scheduling phase}

The scheduling phase will be started periodically after clustering phase. It includes three steps in each period such as cluster head selection, sensor node activity scheduling based Cuckoo Algorithm (CS) optimization and monitoring.

\section{A. Cluster head selection}

After producing the clusters, the exchange of information among the core points (sensor nodes) is achieved inside each cluster, where each core point sends a message to all the core points inside the cluster. It includes the whole essential information like rested power, status, position, members' number, the entire number of devices in the cluster, etc.

In this step, every sensor node inside each cluster will involve the information of other nodes in the same cluster. Therefore, each node in the same cluster will 
achieve the Eq. 7 using the information of each member inside the node. The node that gives the better value of the Eq. 7 will be chosen as a cluster head in the current cluster for this period. The whole devices inside the cluster will perform the same calculation and will produce the same results for the winner device. This will be executed in a distributed way and every node will know if it is a cluster head or not.

$$
\begin{aligned}
& \quad \text { FitVal }_{j}=\frac{E_{\text {remaining }}}{E_{\text {initial }}}+\left(1-\sum_{j \in N}\left|S_{j}(x, y)-S_{i}(x, y)\right|\right)+ \\
& \frac{\left.S_{j} \text { (Members }\right)}{\text { Cluster(Members })}
\end{aligned}
$$

Where E_remaining is the residual energy of the node $j$, E_initial is the initial energy value of node $\mathrm{j}, \mathrm{N}$ is the number of nodes in the current cluster, $\mathrm{S} \_\mathrm{j}(\mathrm{x}, \mathrm{y})$ and $S \_i(x, y)$ refer to the locations of nodes $S \_j$ and $S \_i$ respectively. $S \_j$ (Members) indicates the number of nodes members of node $j$, Cluster (Members) indicates the whole number of nodes in the cluster.

In all the clusters of the WSN, the cluster heads are chosen in an independent, asynchronous, and distributed way.

\section{B. Activity scheduling based Cuckoo Algorithm (CS)}

In this part, the optimization model of the scheduling problem will be formulated, and then a Cuckoo Algorithm will be employed because it is easy to implement and it has fewer tuning parameters.. The Cuckoo Algorithm can solve this model to find the optimal/near optimal solution by producing the best schedule of sensor devices to take the mission of monitoring in the next step in the current period.

Scheduling mathematical model is used to optimize the network lifetime and the coverage of the WSN.

In this paper, two objectives are considered into account during formulating the scheduling optimization model: minimizing the uncovered region inside the cluster region and minimizing the number of active sensor devices after the decision by the CA. This model is inspired from the work in [24] with some modifications by considering decreasing the number of active devices per period as another objective to reduce the consumed energy and enhance the lifespan of WSN. Let parameter A be an indicator for covering the center points of sensor nodes inside each cluster. The Parameter A can be defined as follow:

$$
A j i=\left\{\begin{array}{l}
1 \text { if point center } \mathrm{j} \text { is covered by sensor } \mathrm{i} \\
0 \text { Otherwise }
\end{array}\right.
$$

for $1 \leq \mathrm{j} \leq \mathrm{N}$ and $1 \leq \mathrm{i} \leq \mathrm{N}$, where $\mathrm{N}$ is the number of sensor devices (or centre points) inside the cluster. Let $S$ refers to the solution parameter that can be either 0 or 1 according to the status of the sensor device. It can be defined as follow 


$$
S_{i}=\left\{\begin{array}{l}
1 \text { if Sensor } i \text { is Active } \\
0 \text { Otherwise. }
\end{array}\right.
$$

The coverage probability $\mathrm{Pj}$ of the centre point $\mathrm{j}$ is defined as follows

$$
P_{j}=1-\prod_{i=1}^{N}\left(1-\left(A_{j i} * S_{i}\right)\right)
$$

The first principal objective of the suggested scheduling optimization model is to increase the coverage ratio over the area of interest by decreasing the uncovered ratio $(1-\mathrm{Pj})$ of this area as follows.

$$
P_{j}=1-P_{j}
$$

The second objective is to minimize the number of that cover the same centre point in the sensing field. This can be defined as follows

$$
L_{j}=\left\{\begin{array}{c}
0 \text { if point } j \text { is not covered } \\
\sum_{i=1}^{N}\left(A_{j i} * S_{i}\right)
\end{array}\right.
$$

Hence, the problem of scheduling optimization is modelled as follow.

$$
\begin{array}{ccc}
\operatorname{minimize} & \delta \cdot \sum_{\mathrm{j}=1}^{\mathrm{N}} \mathrm{P}_{\mathrm{j}}+\vartheta \cdot \sum_{\mathrm{j}=1}^{\mathrm{N}} \mathrm{L}_{\mathrm{j}} & \\
\text { Subject to } & & \sum_{i=1}^{N}\left(A_{j i} * S_{i}\right)=1+L_{j}-P_{j} \quad \forall j \in N \text { (15) } \\
& \mathrm{S}_{\mathrm{i}} \in\{0,1\} & \forall \mathrm{i} \in \mathrm{N} \\
P_{j}, L_{j} \geq 0 & \forall j \in N
\end{array}
$$

After that, in each cluster head in the clusters, an energy-efficient activity scheduling mechanism-based CS optimization is achieved. Depending on the sensor nodes centres only, CS optimization yield the ideal cover set of active sensor nodes, which are responsible of sensing throughout the monitoring step in the current period.

We assume the local random walk as follows

$$
x i^{(k+1)}= \begin{cases}x i^{(k)}+r a \text { if } r a>P \alpha \\ x i^{(k)} & \text { otherwise. }\end{cases}
$$

where ra and $r a$ are two random numbers in range $(0,1)$.

As for the suggested algorithm, the assumptions below concerning the sensor nodes will remain constant: 
Sensor coverages take form of circles, where every sensor has a similar coverage with radius Rs.

A sensor is not capable of sensing through or moving across boundaries or obstacles that are regarded to be walls.

The sensing quality remains fixed within Rs and equals zero when outside it (following a binary model).

Algorithm 3 illustrates the Scheduling based CS for providing the optimal or near optimal schedule of sensor devices to stay active in the monitoring step in the current period.

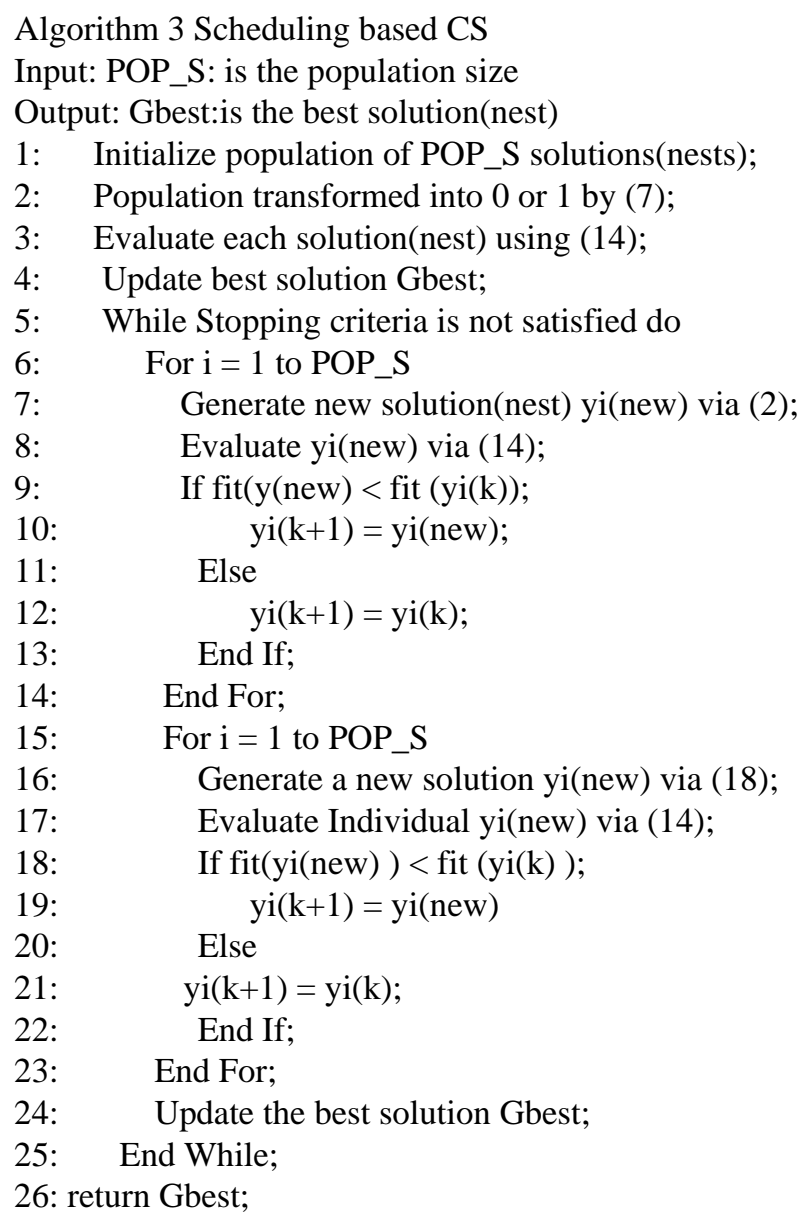

The Scheduling based CS can be identified as an evolutionary algorithm of global optimization whose basis is the distinct breeding behaviour [15] of the cuckoo bird, in addition to the Levy flight, a pattern used by bird for searching food. 
Firstly, a population is initialized which consists of several candidate solutions that are generated in a random manner. These solutions undergo improvement generation after another until the maximal number of generations is reached or a certain condition is satisfied. The improving process which the first solution is subjected to takes place through applying the levy flight on a population, after which the least useful solution is disposed of and the better ones are kept.

The initial population includes a constant number of nests; as it takes form of a matrix with $\mathrm{k}$ rows and $\mathrm{n}$ columns, representing the number of nests and sensors respectively. The Scheduling based CS algorithm can be explained in more details as follows.

- Generating the initial cuckoo Population (nests): The initial population has been created with nests that represent possible sensor schedule solutions for covering the whole cluster region. According to the CS algorithm, the type of the initial population's values will be real.

- Representation of Solution: Given the fact that the aim of the suggested CS is finding the (near) optimum sensor node scheduling that can be responsible of monitoring the cluster region throughout the following step. A nest is identified as a schedule of the sensor nodes, and every nest includes several eggs. Therefore, the egg will include a value 1 that corresponds to the active device or it has value 0 that refers to sleep mode of sensor device. The sigmoid function is used to convert the continues population to discrete population with 0 or 1 .

- Fitness function: Next, all individuals undergo evaluation, as fitness values are assigned based on their fitness functionality presented in Eq. (14). The proposed CS indicates that solutions with minimal fitness values tend to be considered as the optimal candidates. A negative correlation seems to exist between the fitness value and an individual's opportunity to survive. The function tends to reward a decline in the number of sensor nodes covering the same sensor device center, meanwhile a decline to zero is penalized.

- Generate a new nest (solution) xi(k+1): The standard CS makes use of both a global and local random walk combined in a random manner. The former is indicated by Eq. 2, whereas the local random walk is given by Eq. 18 .

- Evaluate New Nests (solutions): each new individual is evaluated using Eq. 14.

- Immigration: As the new individuals (solutions) have been evaluated, the algorithm is iterated through two steps: first, based on the CS algorithm, is replacing all nests (with exception of the ideal one) by a new solution produced through a random walk with Lévy flight around the (so far) optimal nest in terms of quality, and secondly, calculating the $\mathrm{P} \alpha$ fraction of the worst nest so as to replace them by newer ones.

- Update Gbest: The global best is update according to the new best solution that reached by this algorithm in this iteration. 


\section{Monitoring:}

After producing the best schedule of sensor devices by the Scheduling based CS algorithm, the cluster head will send a messages to all sensor devices in the cluster to inform them their status in the next step (monitoring). Each sensor device inside cluster will receive this message. If the message contains 0 , it means that it must stay in sleep mode until the starting the next period. If the message includes 1 , this means that the sensor device must be stay active to perform all the tasks during this step.

\section{Performance Evaluation and Analysis}

In this section, the evaluation is made regarding the efficiency of the CSA technique through executing multiple experiments with the use of $\mathrm{C}++$ custom simulator. Table 1 presents the paramaters applied in the simulating process. Fifty executions have been performed by means of different WSN topologies. The presented results indicate the average rate of these executions. Five network sizes from100 to 300 nodes have been used in the simulation process, deploying nodes in a controlled manner over a sensing area of $(50 \times 25) \mathrm{m} 2$ for ensuring a full coverage for the present area of interest. The suggested protocol uses the energy model discussed in [49].

Table 1 the variables applied in the simulating process.

\begin{tabular}{|c|c|}
\hline Parameter & Value \\
\hline Field of the Sensing & $(50 * 25) \mathrm{m} 2$ \\
\hline WSN size & $100,150,200,250$ and 300 nodes \\
\hline Range of the initial Energy & 500-700 joules \\
\hline Rs & 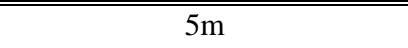 \\
\hline Rc & $10 \mathrm{~m}$ \\
\hline POP_S & 30 \\
\hline 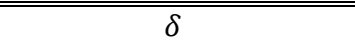 & 0.05 \\
\hline 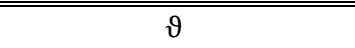 & 0.95 \\
\hline
\end{tabular}


The power of all sensor nodes was initialized in a random manner in the range [500 - 700]. Similar performance metrics which had been applied by [49] have been employed in evaluating the performance of the CSA technique, which include Coverage Ratio, Active Sensors Ratio, Network Lifetime, and Energy Consumption. Additionally, three other methods were used in drawing a comparison, namely DESK [47], GAF [46], and PeCO [49].

\subsection{Coverage Ratio}

The average coverage ratios of each of these 5 methods for 200 nodes are presented in Figure 2. During the first periods, DESK, GAF, and PeCO result in a slightly better coverage rates $(99.99 \%, 99.96 \%$, and $98.76 \%$ respectively), as compared to the $(97.1 \%)$ provided by CSA. The reason behind this is that CSA turns off relatively more redundant nodes than DESK, GAF, and PeCO. After the 65th period, the CSA tends to result in a more favourable coverage performance than the alternative methods, maintaining a coverage rate over $80 \%$ for many rounds. Such an increase in efficiency is the result of the large quantity of energy that has been saved by CSA during the initial rounds.

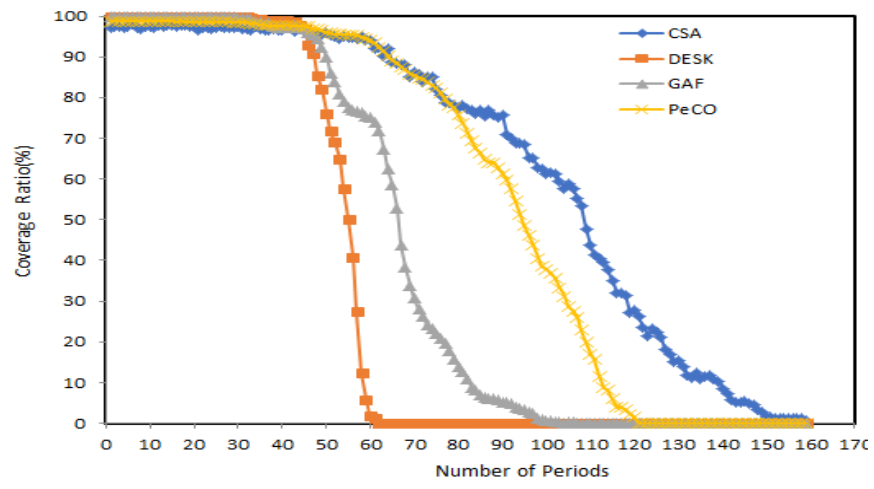

Fig.2: Coverage ratio for WSN size of 200 deployment nodes.

\subsection{Active Sensors Ratio}

Decreasing the number of active nodes during each round is important for conserving more power, hereby maximizing the WSN lifespan. Figure 6 illustrates the average ratio of active nodes for every 200 deployed ones. During the first fifteen periods, DESK, GAF and PeCO activated $30.68 \%$,34.5 \% and 20.18 nodes respectively, whereas CSA activated only $19.8 \%$ sensor nodes. The CSA protocol 
tends to activate more nodes along with the increase in period number, so as to provide an increased coverage rate as shown in Figure 3.

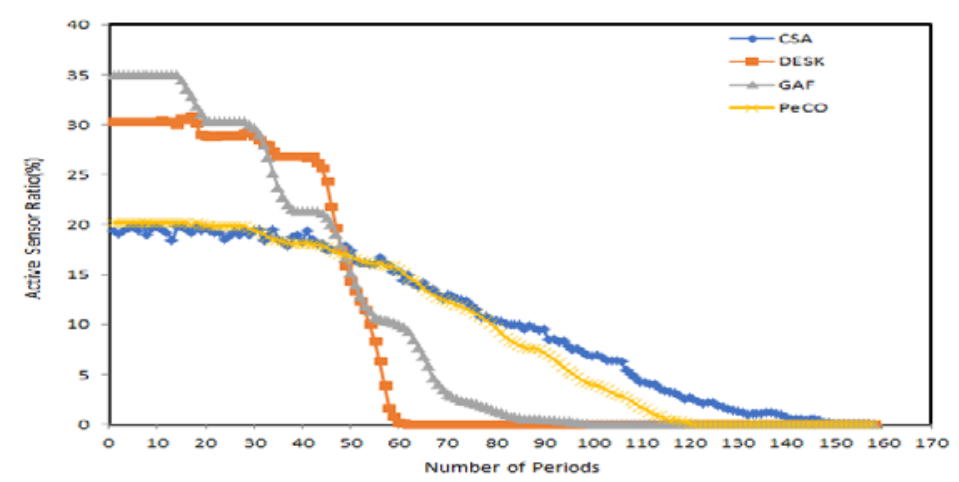

Fig.3: Active sensors ratio for WSN's size of 200 deployed nodes.

\subsection{Energy Consumption}

The current subsection introduces the effect of energy consumption on behalf of the network throughout several statuses of the sensor node (like during the modes of communicating, computing, and listening, as well as the active and sleep statuses), for different WSN sizes. An investigation is made of other approaches being compared. Each of Figure 4 (a) and (b) illustrate the amount of energy consumption for different WSN sizes, in addition to Lifespan95 and Lifespan50. The LifespanX refers to the total time during which the WSN can provide a coverage higher than $\mathrm{X} \%$. The relative superiority of CSA in terms of economizing could be concluded from the illustration. Both Figures indicate the reduction in the amount of energy consumed by CSA in comparison with other methods. The rate of energy consumption hits relatively lower for Lifespan95 and Lifespan50.

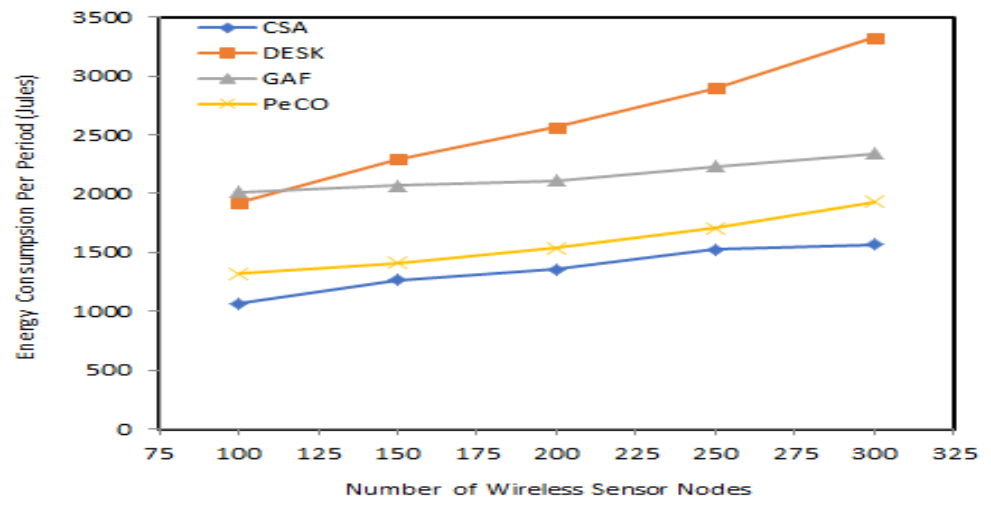

(a) 


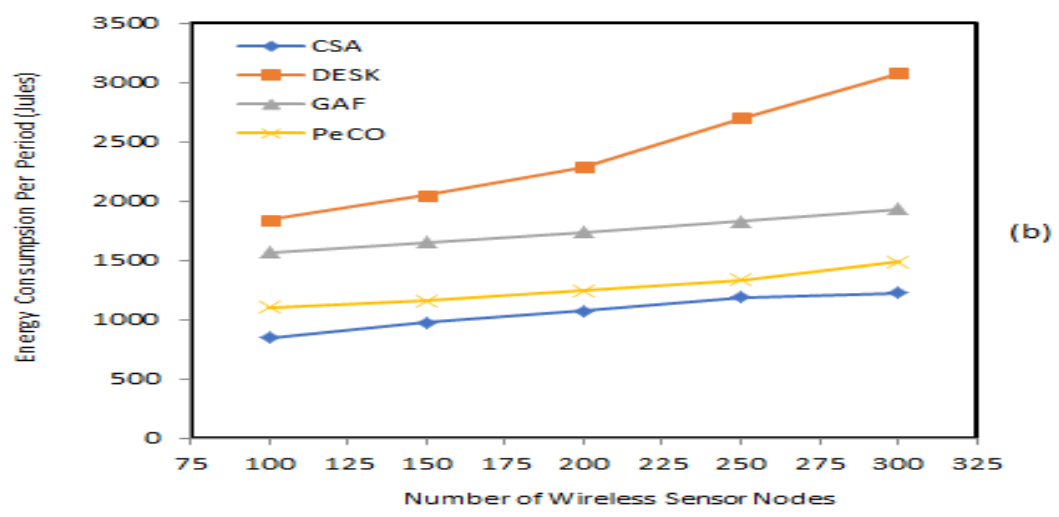

Fig. 4: Energy consumption per round for (a) Lifetime95 and (b) Lifetime50.

\section{Conclusion}

The network lifespan optimization is one of the important factors during designing the WSNs. This paper proposed Cuckoo Scheduling Algorithm (CSA) for Improving Lifespan of Cluster-based WSNs. The CSA technique achieves two phases: clustering and scheduling. First, the WSN is grouped into groups using the DBSCAN method. Second, the scheduling phase is periodic and composed of three steps: cluster head election, scheduling decision-based CA, and covering. The sensor nodes in each cluster determine their cluster head. The selected cluster head performs CA to select the suitable schedule of sensor nodes that take the mission of sense during the current period. The simulation results show that the CSA technique enhances the network lifespan and coverage ratio, and improve the lifespan of WSNs.

\section{References}

[1] Idrees, A. K., \& Al-Qurabat, A. K. M. (2020). Energy-Efficient Data Transmission and Aggregation Protocol in Periodic Sensor Networks Based Fog Computing. Journal of Network and Systems Management, 29(1), 1-24.

[2] Idrees, A. K., Alhussaini, R., \& Salman, M. A. (2020). Energy-efficient two-layer data transmission reduction protocol in periodic sensor networks of IoTs. PERSONAL AND UBIQUITOUS COMPUTING.

[3] Idrees, A. K., Deschinkel, K., Salomon, M., \& Couturier, R. (2018). Multiround distributed lifetime coverage optimization protocol in wireless sensor networks. The Journal of Supercomputing, 74(5), 1949-1972.

[4] Idrees, A. K., Al-Qurabat, A. K. M., Abou Jaoude, C., \& Al-Yaseen, W. L. (2019, June). Integrated divide and conquer with enhanced $k$-means technique for energy-saving data aggregation in wireless sensor networks. In 2019 15th International Wireless Communications \& Mobile Computing Conference (IWCMC) (pp. 973-978). IEEE. 
[5] Guo, J., Karimi-Bidhendi, S., \& Jafarkhani, H. (2020, June). Energy-Efficient Node Deployment in Wireless Ad-hoc Sensor Networks. In ICC 2020-2020 IEEE International Conference on Communications (ICC) (pp. 1-6). IEEE.

[6] Idrees, A. K., Al-Mamory, S. O., \& Couturier, R. (2020, June). Energy-Efficient Particle Swarm Optimization for Lifetime Coverage Prolongation in Wireless Sensor Networks. In International Conference on New Trends in Information and Communications Technology Applications (pp. 200-218). Springer, Cham.

[7] Bouzid, S. E., Serrestou, Y., Raoof, K., Mbarki, M., Omri, M. N., \& Dridi, C. (2020). Wireless sensor network deployment optimisation based on coverage, connectivity and cost metrics. International Journal of Sensor Networks, 33(4), 224-238.

[8] Idrees, A. K., Deschinkel, K., Salomon, M., \& Couturier, R. (2015). Distributed lifetime coverage optimization protocol in wireless sensor networks. The journal of supercomputing, 71(12), 4578-4593.

[9] Hussein, W., \& Idrees, A. K. (2017). Sensor activity scheduling protocol for lifetime prolongation in wireless sensor networks. Kurdistan Journal of Applied Research, 2(3), 7-13..

[10] Idrees, A. K., \& Al-Yaseen, W. L. (2020). Distributed genetic algorithm for lifetime coverage optimization in wireless sensor networks. Int. J. Adv. Intell. Paradigm.

[11] Jia, S. G., Lu, L. P., Su, L. D., Xing, G. L., \& Zhai, M. Y. (2013). An efficient sleeping scheduling for save energy consumption in wireless sensor networks. Advanced Materials Research, 756(759), 2288-2293.

[12] Chen, C. P., Mukhopadhyay, S. C., Chuang, C. L., Liu, M. Y., \& Jiang, J. A. (2015). Efficient coverage and connectivity preservation with load balance for wireless sensor networks. IEEE Sensors Journal, 15(1), 48-62.

[13] Hao, J., Zhang, B., Jiao, Z., \& Hashemi, M. R. (2015). An adaptive compressive sensing-based sample scheduling mechanism for wireless sensor networks. Pervasive and Mobile Computing, 2, 113-125.

[14] Silberschatz, A., Galvin, P. B., \& Gagne, G. (1998). Operating system concepts. Boston: Addison-Wesley.

[15] Yuan, X., \& Duan, Z. (2009). Fair round-robin: A low complexity packet scheduler with proportional and worst-case fairness. IEEE Transactions on Computers, 58(3), 365379.

[16] Yaxiong Z, Jie W. Stochastic sleep scheduling for large scale wireless sensor networks. In: 2010 IEEE international conference on communications (ICC); 23-27 May 2010. p.1-5.

[17] Chih-fan H, Mingyan L. Network coverage using low duty-cycled sensors: random \& coordinated sleep algorithms. In: IPSN 2004. Third international symposium on information processing in sensor networks; 26-27 April 2004. p. 433-42.

[18] Nath S, Gibbons PB. Communicating via fireflies: geographic routing on duty-cycled sensors. In: IPSN 2007. Sixth international symposium on information processing in sensor networks; 25-27 April 2007. p. 440-9.

[19] Zhuxiu Y, Lei W, Lei S, Hara T, Zhenquan Q. A balanced energy consumption sleep scheduling algorithm in wireless sensor networks. In: Seventh international conference on wireless communications and mobile computing conference (IWCMC); 4-8 July 2011. p. 831-5.

[20] Chih-Fan H, Mingyan L. Network coverage using low duty- cycled sensors: Random and coordinated sleep algorithms. IPSN 3rd International Symposium on Information Processing in Sensor Networks; 2004 Apr 26-27. p. 433-42.

[21] Yaxiong Z, Jie W. Stochastic sleep scheduling for large scale wireless sensor networks. 2010 IEEE InternationalConference on Communications (ICC); 2010 May 23-27. p. $1-5$. 
[22] Sun LJ, Wei J, Guo J, et al. Node scheduling algorithm for heterogeneous wireless sensor networks. Acta Electron Sinica 2014; 42(10): 1907-1912.

[23] Ahmed et al, "Sleep-awake energy efficient distributed clustering algorithm for wireless sensor networks", Computers and Electrical Engineering, pp 1-14, 2015.

[24] Sander, Jörg; Ester, Martin; Kriegel, Hans-Peter; Xu, Xiaowei (1998). "Density-Based Clustering in Spatial Databases: The Algorithm GDBSCAN and Its Applications". Data Mining and Knowledge Discovery. Berlin: Springer-Verlag. 2 (2): 169-194.

[25] Xu, Y., Heidemann, J., Estrin, D. (2001): Geography-informed energy conservation for ad hoc routing.In: Proceedings of the 7th Annual International Conference on Mobile Computing and Networking, pp. 70-84. ACM

[26] Yu, J., Ren, S., Wan, S., Yu, D., Wang, G. (2012): A stochastic k-coverage scheduling algorithm in wireless sensor networks. Int. J. Distrib. Sens. Netw. 8(11), 746501

[27] Idrees, A.K., Deschinkel, K., Salomon, M. Raphael Couturier (2015): Distributed lifetime coverage optimization protocol in wireless sensor networks. J Supercomput 71, 4578-4593.

[28] Idrees, A. K., Deschinkel, K., Salomon, M., \& Couturier, R. (2016). Perimeter-based coverage optimization to improve lifetime in wireless sensor networks. Engineering Optimization, 48(11), 1951-1972.

[29] Mazin Kadhum Hameed and Ali Kadhum Idrees (2020). Distributed Clustering-based DBSCAN Protocol for Energy Saving in IoT Networks, 2nd International Conference on Communication, Computing and Electronics Systems (ICCCES 2020) on 21-22, October 2020, Lecture Notes in Electrical Engineering Series, ISSN: 1876-1100, Springer. 